\title{
Copper-Catalyzed SyNTHESIS OF VinYL SULFIDES
}

\author{
Craig G. Bates, Pranorm Saejueng, Michael Q. Doherty and D. Venkataraman* \\ Department of Chemistry, University of Massachusetts Amherst, 710 N. Pleasant St., Amherst, MA 01003.
}

\section{SUPPORTING INFORMATION}

General. All of the reactions reported herein were conducted under an inert atmosphere of argon in oven-dried glassware. All reagents and solvents were obtained from Acros, Alfa Aesar or from Aldrich and were used without further purification. Potassium Phosphate (Alfa Aesar, 97\%) was stored in an argon filled glove box. All vinyl iodides used in this paper have been synthesized using procedures previously reported in the literature. ${ }^{1-4}$ Purification was performed by flash chromatography using standard grade silica gel, 230-450 mesh or activated neutral aluminum oxide 50-200 micron. The yields given refer to isolated yields of the characterized compounds, deemed pure by elemental analyses, ${ }^{1} \mathrm{H}$ NMR and ${ }^{13} \mathrm{C}$ NMR. In certain cases GC yields were reported. All GC yields were calculated using $n$-dodecane as an internal standard; the correction factors used to calculate the product yields were determined using an analytically pure sample. The Gas Chromatograph used was a Hewlett Packard 6850 GC series with a 30-meter HP-1 100\% dimethylpolysiloxane capillary column. NMR spectra were recorded on a Bruker AVANCE $400 \mathrm{MHz}$ spectrometer. Chemical shifts were reported for the major isomer in parts per million $(\delta)$. The peak patterns are indicated as follows: $\mathrm{s}$, singlet; d, doublet; t, triplet; dd, doublet of doublets; dt, doublet of a triplet; td, triplet of a doublet; sept, septet; m, multiplet; and q, quartet. The coupling constants, J, are reported in Hertz $(\mathrm{Hz})$. TMS was used as the internal reference. Infrared spectra were recorded using a Midac MD1200-SP3 and are reported in reciprocal centimeters $\left(\mathrm{cm}^{-1}\right)$ along with relative intensity ( $\mathrm{w}=$ weak, $\mathrm{m}=$ medium, $\mathrm{s}=$ strong absorption). Elemental analyses were performed at the Microanalysis Laboratory, University of Massachusetts Amherst by Dr. Greg Dabkowski. The reported melting points were uncorrected. 


\section{SYNTHESIS OF COPPER(I) COMPLEXES}

The following copper(I) complexes were synthesized using procedures outlined in literature: Tris(triphenylphosphine)copper(I) bromide, ${ }^{5} \quad\left[\mathrm{Cu}\left(\mathrm{CH}_{3} \mathrm{CN}\right)_{4}\right] \mathrm{PF}_{6}{ }^{6}{ }^{6}$

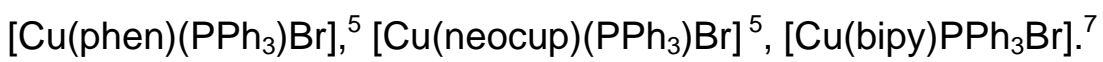

\section{SYNTHESIS OF COPPER(I) COMPLEXES}

Nitratobis(triphenylphosphine)copper(I): In an Erlenmeyer flask equipped with a Teflon-coated stir bar, methanol $(100 \mathrm{~mL})$ was heated to boiling and triphenylphosphine (Alfa Aesar, $24.22 \mathrm{~g}, 92.34 \mathrm{mmol}$ ) was slowly added to the stirring methanol. After the complete dissolution of triphenylphosphine, $\mathrm{Cu}\left(\mathrm{NO}_{3}\right)_{2} \cdot 2.5 \mathrm{H}_{2} \mathrm{O}$ (Fisher Scientific, $7.16 \mathrm{~g}$, $30.78 \mathrm{mmol}$ ) was added in small portions. No special precautions were taken for the exclusion of air. Upon addition of the copper(II) nitrate, a white precipitate formed. After the completion of the addition, the contents were stirred for 30 minutes and the flask was allowed to cool to ambient temperature. The reaction mixture was then filtered through a Buchner funnel and the white residue was washed repeatedly with ethanol and then with diethyl ether. The resultant white solid was dried under dynamic vacuum to give $\mathrm{Cu}\left(\mathrm{PPh}_{3}\right)_{2} \mathrm{NO}_{3}\left(12.378 \mathrm{~g}, 62 \%\right.$ yield). m.p. $-238-240{ }^{\circ} \mathrm{C}$. The cell constants, contents and the space group are identical to that of the already reported structure of $\mathrm{Cu}\left(\mathrm{PPh}_{3}\right)_{2} \mathrm{NO}_{3}$ (Cambridge Structural Database Refcode-NITPPC01).

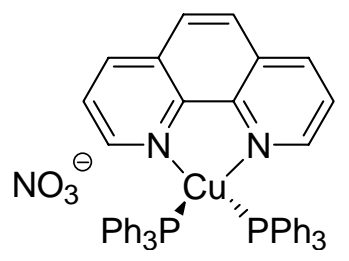

[Cu(phen)( $\left.\left(\mathrm{PPh}_{3}\right)_{2}\right] \mathrm{NO}_{3}$ : In an Erlenmeyer flask equipped with a Teflon-coated magnetic stir bar, Nitratobis(triphenylphosphine)copper(I) (977 mg, $1.50 \mathrm{mmol}$ ) was added to chloroform (20 mL). After complete dissolution, triphenylphosphine (393 mg, $1.50 \mathrm{mmol})$, followed by 1,10-phenanthroline $(270 \mathrm{mg}, 1.50 \mathrm{mmol}$ ) was then added. The colorless solution immediately turned yellow. The contents of the flask were allowed to stir for 30 
minutes at room temperature. Afterwards the solvent was removed in vacuo to afford a yellow solid. Recrystallization was achieved by vapor diffusion of diethyl ether into a solution of the solid dissolved in $30 \mathrm{~mL}$ of dichloromethane (931 mg, 75\% yield). m.p. 202-204 ${ }^{\circ} \mathrm{C}$.

\section{ACTIVITY OF COPPER CATALYSTS:}

Figure 1. A comparison of well-defined copper(I) complexes, copper(I) salts and additives as catalysts for the crosscoupling of thiophenol and $(E)$-1-iodooctene. The lines drawn are for visual reference only.

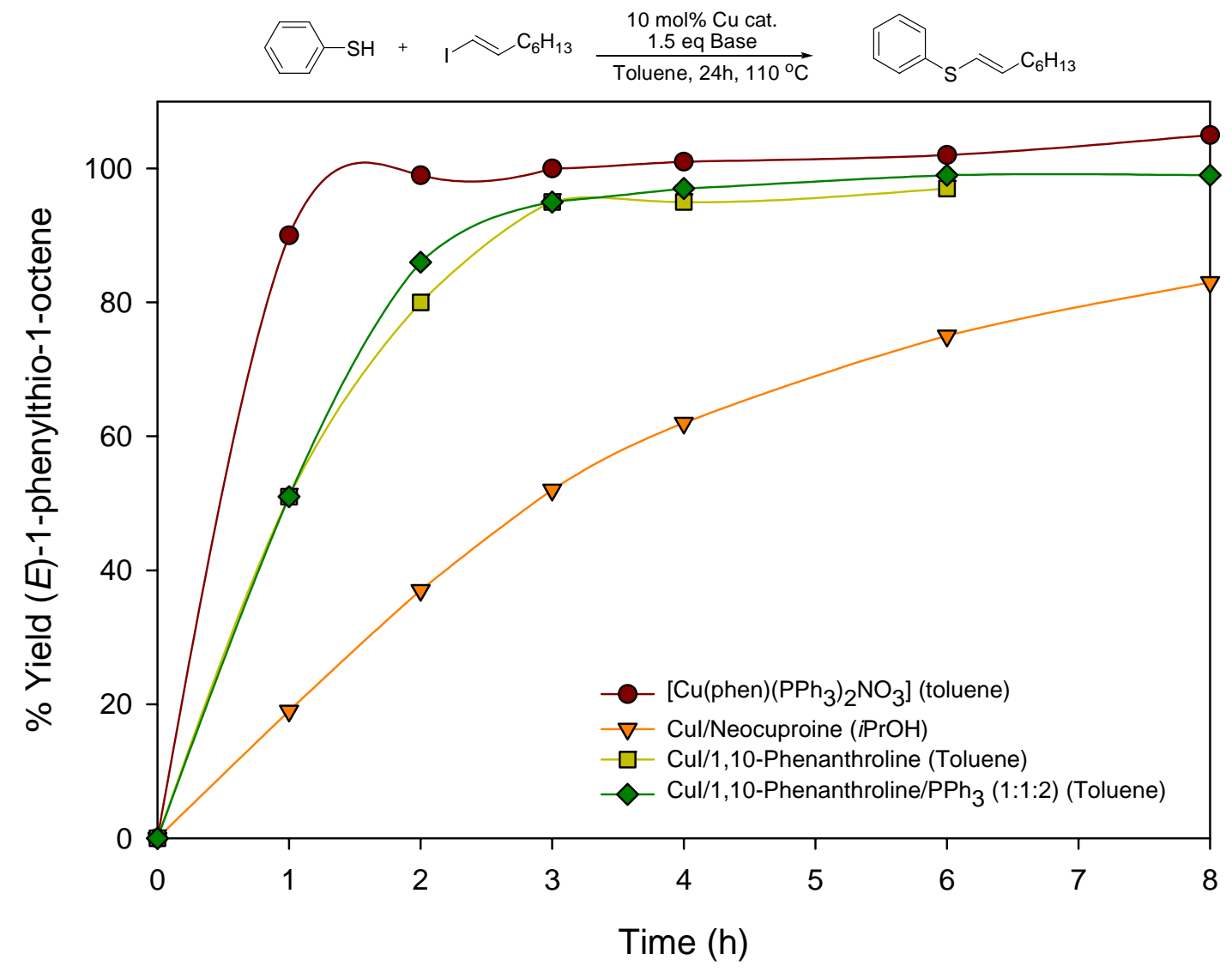




\section{OPTIMIZATION OF BASE:}

Table 1. A comparison of various bases for the cross-coupling of thiophenol and $(E)$-1-iodooctene using $10 \mathrm{~mol} \%$ $\left[\mathrm{Cu}\right.$ (neocup) $\left.\mathrm{PPh}_{3} \mathrm{Br}\right]$ as the catalyst in toluene for 24 hours

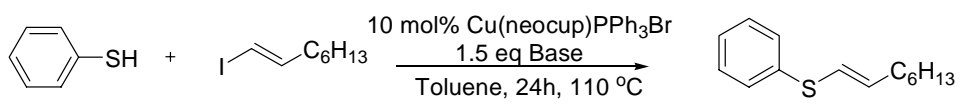

\begin{tabular}{ccc} 
& \multicolumn{1}{c}{$\mathrm{GC}$} & \\
Base $(1.5 \mathrm{eq})$ & Yield & $\mathrm{E} / \mathrm{Z}$ \\
\hline $\mathrm{K}_{3} \mathrm{PO}_{4}$ & $99 \%$ & $15: 1$ \\
$\mathrm{~K}_{2} \mathrm{CO}_{3}$ & $99 \%$ & $10: 1$ \\
$\mathrm{Cs}_{2} \mathrm{CO}_{3}$ & $96 \%$ & $4: 1$ \\
$\mathrm{CsOAc}$ & $94 \%$ & $5: 1$ \\
$\mathrm{DBU}$ & $86 \%$ & $15: 1$ \\
$\mathrm{Na}_{2} \mathrm{CO}$ & $84 \%$ & $3: 1$ \\
$\mathrm{NaOtBu}$ & $83 \%$ & $19: 1$ \\
$\mathrm{KOtBu}$ & $47 \%$ & $>25: 1$
\end{tabular}

\section{SYNTHESIS OF VINYL SULFIDES:}

General Procedure: In an argon-filled glove box, a Pyrex glass tube $(2.5 \mathrm{~cm}$ in diameter) equipped with a Teflon-coated stir bar, was charged with potassium phosphate (Alfa Aesar, $0.6368 \mathrm{~g}, 3.00 \mathrm{mmol}$ ) and [Cu(phen) $\left.\left(\mathrm{PPh}_{3}\right)_{2}\right] \mathrm{NO}_{3}(.0831 \mathrm{~g}, 5.0$ mol\%). The tube was then sealed with a rubber septum, taken out of the glove box and toluene $(4.0 \mathrm{~mL})$ and $2.00 \mathrm{mmol}$ of the appropriate thiol and $2.00 \mathrm{mmol}$ of the appropriate vinyl iodide were injected into the tube through the septum. The contents were then stirred at $110{ }^{\circ} \mathrm{C}$ for 4 hours unless specified otherwise. The reaction mixture was then cooled to room temperature and filtered through a pad of celite to remove any insoluble residues and the pad of celite was washed with $50 \mathrm{~mL}$ of ethyl acetate. The filtrate was concentrated in vacuo; the residue was purified by flash column chromatography on silica gel or neutral aluminum oxide to obtain the analytically pure product. 


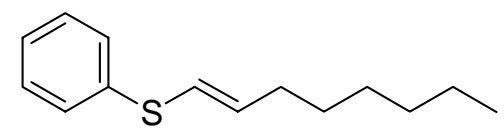

(E)-1-phenylthio-1-octene (Table 2, entry 1): The general procedure was used to convert thiophenol and $(E)$-1-iodooctene to the title product. Purification by flash chromatography (silica gel) (3\% triethylamine in hexanes as the eluent) gave the analytically pure product as a colorless liquid (407 mg, 93\% yield). ${ }^{1} \mathrm{H}$ NMR (400 MHz, $\left.\mathrm{CDCl}_{3}\right) \delta 7.31-7.27(\mathrm{~m}, 4 \mathrm{H}), 7.16(\mathrm{~m}, 1 \mathrm{H}), 6.14(\mathrm{td}, \mathrm{J}=14.9,2.4 ; 1 \mathrm{H}), 5.98(\mathrm{td}, \mathrm{J}=15.0$, $6.8 \mathrm{~Hz} ; 1 \mathrm{H}), 2.16(\mathrm{~m}, \mathrm{~J}=8.1,7.0,1.2 \mathrm{~Hz} ; 2 \mathrm{H}), 1.42-1.29(\mathrm{~m}, 8 \mathrm{H}), 0.89(\mathrm{t}, \mathrm{J}=6.8 \mathrm{~Hz} ; 3 \mathrm{H})$. ${ }^{13} \mathrm{C}$ NMR $\left(100 \mathrm{MHz}, \mathrm{CDCl}_{3}\right) \delta 137.84,136.68,128.86,128.30,125.92,122.47,33.07$, 31.62, 28.94, 28.76, 22.60, 14.07. Anal. Calc'd. for $\mathrm{C}_{14} \mathrm{H}_{20} \mathrm{~S}$ : C - 76.30; H - 9.15; S 14.58 Found, C - 76.31; H - 9.16; S - 14.58.

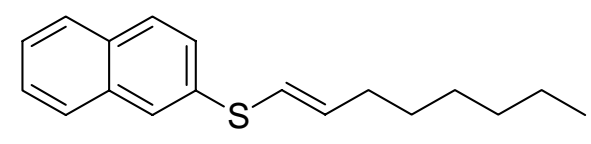

(E)-naphthalen-2-yl(oct-1-enyl)sulfane (Table 2, entry 2): The general procedure was used to convert 2-naphthalenethiol and $(E)$-1-iodooctene to the title product. Purification by flash chromatography (silica gel) (3\% triethylamine in hexanes as the eluent) gave the analytically pure product as a colorless liquid (526 mg, 97\% yield). ${ }^{1} \mathrm{H}$ NMR (400 $\left.\mathrm{MHz}, \mathrm{CDCl}_{3}\right) \delta$ 7.78-7.71 (m, 4H), 7.47-7.37 (m, 3H), $6.21(\mathrm{td}, \mathrm{J}=14.9,1.2 \mathrm{~Hz} ; 1 \mathrm{H}), 6.05$ $(\mathrm{td}, \mathrm{J}=14.9,6.8 \mathrm{~Hz} ; 1 \mathrm{H}), 2.18(\mathrm{~m}, \mathrm{~J}=7.7,6.8,1.1 \mathrm{~Hz} ; 2 \mathrm{H}), 1.44-1.30(\mathrm{~m}, 8 \mathrm{H}), 0.90(\mathrm{t}, \mathrm{J}=$ $6.6 \mathrm{~Hz} ; 3 \mathrm{H}) .{ }^{13} \mathrm{C}$ NMR $\left(100 \mathrm{MHz}, \mathrm{CDCl}_{3}\right) \delta 138.28,134.15,133.75,131.74,128.38$, $127.69,127.03,126.58,126.50,126.13,125.56,120.38,33.13,31.64,28.95,28.78$, 22.63, 14.10. Anal. Calc'd. for $\mathrm{C}_{18} \mathrm{H}_{22} \mathrm{~S}$ : C - 79.94; H - 8.20; S - 11.86; Found, C - 79.93; $\mathrm{H}-8.05$; S - 11.86 .

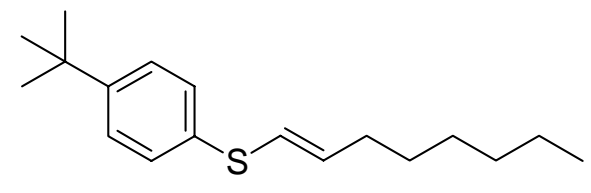

(E)-(4-tert-butylphenyl)(oct-1-enyl)sulfane (Table 2, entry 3): The general procedure was used to convert 4-tert-butylthiophenol and $(E)$-1-iodooctene to the title product. 
Purification by flash chromatography (silica gel) (3\% triethylamine in hexanes as the eluent) gave the analytically pure product as a colorless liquid (509 mg, 92\% yield). ${ }^{1} \mathrm{H}$ NMR (400 MHz, $\left.\mathrm{CDCl}_{3}\right) \delta$ 7.33-7.24 (m, 4H), 6.11 (td, J= 15.0, $\left.1.2 \mathrm{~Hz} ; 1 \mathrm{H}\right), 5.94$ (td, J= 14.9, $6.8 \mathrm{~Hz} ; 1 \mathrm{H}), 2.14(\mathrm{~m}, \mathrm{~J}=7.8,6.9 .1 .0 \mathrm{~Hz} ; 2 \mathrm{H}), 1.45-1.29(\mathrm{~m}, 17 \mathrm{H}), 0.89$ (t, J=6.6 $\mathrm{Hz} ; 3 \mathrm{H}) .{ }^{13} \mathrm{C}$ NMR $\left(100 \mathrm{MHz}, \mathrm{CDCl}_{3}\right) \delta 149.37,136.79,132.99,128.65,126.01,121.35$, $34.50,33.11,31.70,31.33,29.06,28.83,22.67,14.15$. Anal. Calc'd. for $\mathrm{C}_{18} \mathrm{H}_{28} \mathrm{~S}$ : C 78.04; H - 10.11; S - 11.60 Found, C - 78.19; H - 10.21; S - 11.60.

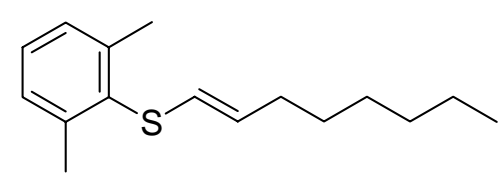

(E)-(2,6-dimethylphenyl)(oct-1-enyl)sulfane (Table 2, entry 4): The general procedure was used to convert 2,6-dimethylthiophenol and $(E)$-1-iodooctene to the title product. Purification by flash chromatography (silica gel) (3\% triethylamine in hexanes as the eluent) gave the analytically pure product as a colorless liquid (493 mg, 99\% yield). ${ }^{1} \mathrm{H}$ NMR (400 MHz, $\left.\mathrm{CDCl}_{3}\right) \delta 7.13(\mathrm{~m}, 3 \mathrm{H}), 5.79(\mathrm{td}, \mathrm{J}=14.9,1.3 ; 1 \mathrm{H}), 5.23(\mathrm{td}, \mathrm{J}=14.8,7.0$ $\mathrm{Hz} ; 1 \mathrm{H}), 2.47(\mathrm{~s}, 6 \mathrm{H}), 2.14(\mathrm{~m}, \mathrm{~J}=7.7,7.0,1.2 \mathrm{~Hz} ; 2 \mathrm{H}), 1.45-1.29(\mathrm{~m}, 17 \mathrm{H}), 0.89(\mathrm{t}, \mathrm{J}=$ $6.6 \mathrm{~Hz} ; 3 \mathrm{H}) .{ }^{13} \mathrm{C} \mathrm{NMR}\left(100 \mathrm{MHz}, \mathrm{CDCl}_{3}\right) \delta 149.37,136.79,132.99,128.65,126.01$, $121.35,34.50,33.11,31.70,31.33,29.06,28.83,22.67,14.15$. Anal. Calc'd. for $\mathrm{C}_{18} \mathrm{H}_{28} \mathrm{~S}$ : C - 78.04; H - 10.11; S - 11.60 Found, C - 78.19; H - 10.21; S - 11.60.

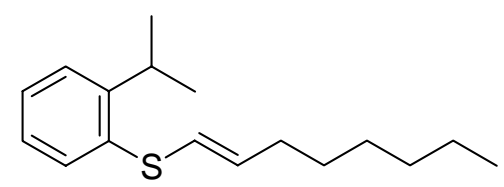

(E)-(2-isopropylphenyl)(oct-1-enyl)sulfane (Table 2, entry 5): The general procedure was used to convert 2-isopropylthiophenol and $(E)$-1-iodooctene to the title product. Purification by flash chromatography (silica gel) (3\% triethylamine in hexanes as the eluent) gave the analytically pure product as a colorless liquid (511 mg, 97\% yield). ${ }^{1} \mathrm{H}$ NMR (400 MHz, $\left.\mathrm{CDCl}_{3}\right) \delta$ 7.32-7.12 (m, 4H), $6.06(\mathrm{td}, \mathrm{J}=14.9,1.3 ; 1 \mathrm{H}), 5.90(\mathrm{~m}, 1 \mathrm{H})$, 3.40 (sept., $1 \mathrm{H}), 2.15(\mathrm{~m}, 2 \mathrm{H})$ 1.42-1.22 (m, $14 \mathrm{H}), 0.88(\mathrm{t}, \mathrm{J}=6.8 \mathrm{~Hz} ; 3 \mathrm{H}) .{ }^{13} \mathrm{C}$ NMR (100 $\left.\mathrm{MHz}, \mathrm{CDCl}_{3}\right) \delta 147.67,136.77,134.25,129.48,126.68,126.26,125.42,121.27,33.11$, 
$31.64,30.22,29.02,28.76,23.23,22.61,14.08$. Anal. Calc'd. for $\mathrm{C}_{17} \mathrm{H}_{26} \mathrm{~S}$ : C - 77.80; H 9.99; S - 12.22 Found, C - 77.62; H - 9.92; S - 12.17.

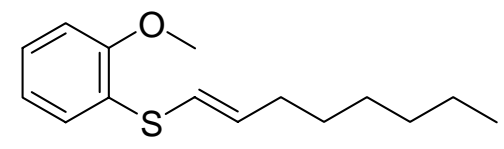

(E)-(2-methoxyphenyl)(oct-1-enyl)sulfane (Table 2, entry 6): The general procedure was used to convert 2-methoxythiophenol and $(E)$-1-iodooctene to the title product. Purification by flash chromatography (silica gel) (5\% ethyl acetate in a $3 \%$ triethylamine in hexanes solution as the eluent) gave the analytically pure product as a colorless liquid (471 mg, 94\% yield). ${ }^{1} \mathrm{H}$ NMR (400 MHz, $\left.\mathrm{CDCl}_{3}\right) \delta 7.22$ (dd, J= 7.8, $\left.1.5 \mathrm{~Hz} ; 1 \mathrm{H}\right), 7.16(\mathrm{~m}$, 1H), $6.90(\mathrm{~m}, 2 \mathrm{H}), 6.09$ (d, J= $15.2 \mathrm{~Hz} ; 1 \mathrm{H}), 6.03$ (td, J= 14.9, $6.2 \mathrm{~Hz} ; 1 \mathrm{H}), 3.87(\mathrm{~s}, 3 \mathrm{H})$, $2.16(\mathrm{~m}, 2 \mathrm{H}), 1.43-1.29(\mathrm{~m}, 8 \mathrm{H}), 0.89(\mathrm{t}, \mathrm{J}=6.5 \mathrm{~Hz}, 3 \mathrm{H}) .{ }^{13} \mathrm{C}$ NMR $\left(100 \mathrm{MHz}, \mathrm{CDCl}_{3}\right) \delta$ 156.09 , 138.64, 128.20, 126.80, 125.20, 121.10, 119.33, 110.36, 55.71, 33.11, 31.59, 28.90, 28.71, 22.57, 14.04. Anal. Calc'd. for $\mathrm{C}_{15} \mathrm{H}_{22} \mathrm{OS}$ : C - 71.95; H - 8.86; S - 12.81 Found, C - 72.20; H - 8.92; S - 12.73.

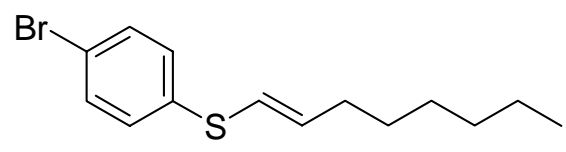

(E)-(4-bromophenyl)(oct-1-enyl)sulfane (Table 2, entry 7): The general procedure was used to convert 4-bromothiophenol and $(E)$-1-iodooctene to the title product. Purification by flash chromatography (silica gel) (3\% triethylamine in hexanes as the eluent) gave the analytically pure product as a colorless liquid (575 mg, 96\% yield). ${ }^{1} \mathrm{H}$ $\operatorname{NMR}\left(400 \mathrm{MHz}, \mathrm{CDCl}_{3}\right) \delta 7.38(\mathrm{td}, \mathrm{J}=8.5,2.0 \mathrm{~Hz} ; 2 \mathrm{H}), 7.14(\mathrm{td}, \mathrm{J}=8.5,1.9 \mathrm{~Hz} ; 2 \mathrm{H}), 6.06$ $(\mathrm{d}, \mathrm{J}=14.9 \mathrm{~Hz} ; 1 \mathrm{H}), 6.0(\mathrm{~m}, 1 \mathrm{H}), 2.16(\mathrm{~m}, 2 \mathrm{H}), 1.44-1.29(\mathrm{~m}, 8 \mathrm{H}), 0.89(\mathrm{t}, \mathrm{J}=6.7 \mathrm{~Hz}, 3 \mathrm{H})$. ${ }^{13} \mathrm{C}$ NMR $\left(100 \mathrm{MHz} \mathrm{CDCl}_{3}\right) \delta 139.12,136.06,131.86,129.63,119.75,119.64,33.07$, $31.60,28.87,28.76,22.60,14.07$. Anal. Calc'd. for $\mathrm{C}_{14} \mathrm{H}_{19} \mathrm{BrS}$ : C - 56.19; H - 6.40; $\mathrm{Br}-$ 26.70; S - 10.71 Found, C - 56.41; H - 6.45; Br - 26.90; S - 10.56. 


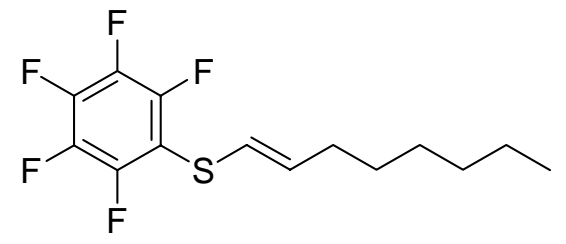

(E)-oct-1-enyl(perfluorophenyl)sulfane (Table 2, entry 8): The general procedure was used to convert pentafluorothiophenol and $(E)$-1-iodooctene to the title product. Purification by flash chromatography (silica gel) (2\% triethylamine in hexanes as the eluent) gave the analytically pure product as a colorless liquid (608 mg, 98\% yield). ${ }^{1} \mathrm{H}$ NMR (400 MHz, CDCl $)_{3} \delta 5.96(\mathrm{~m}, 2 \mathrm{H}), 2.08(\mathrm{~m}, 2 \mathrm{H}), 1.32(\mathrm{~m}, 8 \mathrm{H}), 0.88(\mathrm{t}, \mathrm{J}=6.4 \mathrm{~Hz}$; $3 \mathrm{H}) .{ }^{13} \mathrm{C}$ NMR $\left(100 \mathrm{MHz}, \mathrm{CDCl}_{3}\right) \delta 138.51,117.98,32.79,31.58,28.67,28.64,22.56$, 13.98. Anal. Calc'd. for $\mathrm{C}_{14} \mathrm{H}_{15} \mathrm{~F}_{5} \mathrm{~S}$ : C - 54.18; H - 4.87; S - 10.33 Found, C - 54.12; H 4.80; S - 10.50 .

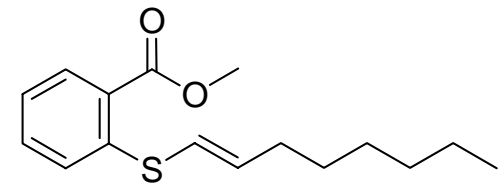

(E)-methyl 2-(oct-1-enylthio)benzoate (Table 2, entry 9): The general procedure was used to convert methyl thiosalicylate and $(E)$-1-iodooctene to the title product. Purification by flash chromatography (neutral alumina) (5\% ethyl acetate in hexanes as the eluent) gave the analytically pure product as a colorless liquid (524 mg, 94\% yield). ${ }^{1} \mathrm{H}$ NMR $\left(400 \mathrm{MHz}, \mathrm{CDCl}_{3}\right) \delta 7.96(\mathrm{dd}, \mathrm{J}=7.8,1.5 \mathrm{~Hz} ; 1 \mathrm{H}), 7.44-7.34(\mathrm{~m}, 2 \mathrm{H}), 7.16(\mathrm{~m}$, $1 \mathrm{H}), 6.33(\mathrm{td}, \mathrm{J}=14.9,6.6 \mathrm{~Hz} ; 1 \mathrm{H}), 6.13(\mathrm{~d}, \mathrm{~J}=15.0 \mathrm{~Hz} ; 1 \mathrm{H}), 3.91(\mathrm{~s}, 3 \mathrm{H}), 2.23$ (q, J= 6.7 $\mathrm{Hz} ; 2 \mathrm{H}), 1.48-1.30(\mathrm{~m}, 8 \mathrm{H}) ; 0.90(\mathrm{t}, \mathrm{J}=6.8 \mathrm{~Hz} ; 3 \mathrm{H}) .{ }^{13} \mathrm{C} \mathrm{NMR}\left(100 \mathrm{MHz}, \mathrm{CDCl}_{3}\right) \delta$ $166.65,142.20,132.20,131.13,126.72,126.57,124.16,119.65,52.01,33.21,31.57$, 28.77, 28.73, 22.57, 14.01. Anal. Calc'd. for $\mathrm{C}_{16} \mathrm{H}_{22} \mathrm{O}_{2} \mathrm{~S}$ : C - 69.02; H - 7.96; S - 11.52 Found, C - 68.97; H - 7.95; S - 11.75.

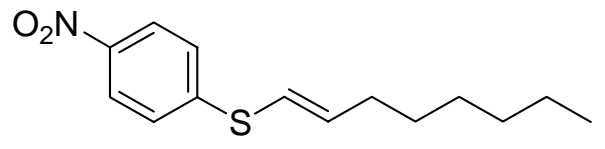


(E)-(4-nitrophenyl)(oct-1-enyl)sulfane (Table 2, entry 10): The general procedure was used to convert 4-nitrothiophenol and $(E)$-1-iodooctene to the title product. Purification by flash chromatography (silica gel) (5\% ethyl acetate in a $3 \%$ triethylamine in hexanes solution as the eluent) gave the analytically pure product as a yellow liquid (494 $\mathrm{mg}$, 93\% yield). ${ }^{1} \mathrm{H}$ NMR (400 MHz, $\mathrm{CDCl}_{3}$ ) $\delta 8.12(\mathrm{td}, \mathrm{J}=8.9,2.0 \mathrm{~Hz} ; 2 \mathrm{H}), 7.32$ (td, $\mathrm{J}=9.0$, $2.5 \mathrm{~Hz} ; 2 \mathrm{H}), 6.25(\mathrm{~m}, 1 \mathrm{H}), 6.13(\mathrm{~d}, \mathrm{~J}=14.9 \mathrm{~Hz} ; 1 \mathrm{H}), 2.25(\mathrm{~m}, 2 \mathrm{H}), 1.50-1.31(\mathrm{~m}, 8 \mathrm{H})$, $0.91(\mathrm{t}, \mathrm{J}=6.8 \mathrm{~Hz}, 3 \mathrm{H}) .{ }^{13} \mathrm{C} \mathrm{NMR}\left(100 \mathrm{MHz}, \mathrm{CDCl}_{3}\right) \delta 147.74,145.16,143.78,125.94$, 123.85, 116.74, 33.17, 31.52, 28.72, 28.61, 22.53, 14.00. Anal. Calc'd. for $\mathrm{C}_{14} \mathrm{H}_{19} \mathrm{NO}_{2} \mathrm{~S}$ : C - 63.36; H - 7.22; N - 5.28; S - 12.08 Found, C - 63.24; H - 7.15; N - 5.42; S - 11.93.

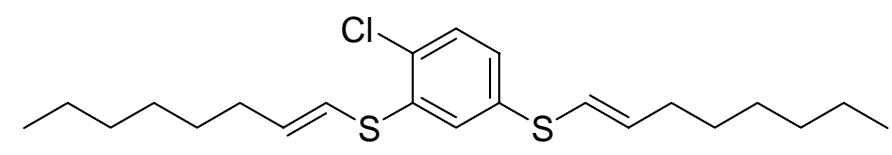

1-chloro-2,4-bis((E)-oct-1-enylthio)benzene (Table 2, entry 11): The general procedure was used to convert 3-chloro-1,3-benzenedithiol and $(E)$-1-iodooctene to the title product. Purification by flash chromatography (silica gel) (3\% triethylamine in hexanes as the eluent) gave the analytically pure product as a colorless liquid (722 $\mathrm{mg}$, 91\% yield). ${ }^{1} \mathrm{H}$ NMR (400 MHz, $\left.\mathrm{CDCl}_{3}\right) \delta 7.17(\mathrm{~m}, 2 \mathrm{H}), 7.00(\mathrm{~m}, 1 \mathrm{H}), 6.10(\mathrm{~m}, 4 \mathrm{H}), 2.18$ $(\mathrm{m}, 4 \mathrm{H}), 1.43-1.30(\mathrm{~m}, 16 \mathrm{H}), 0.89(\mathrm{~m}, 6 \mathrm{H}) .{ }^{13} \mathrm{C}$ NMR $\left(150 \mathrm{MHz}, \mathrm{CDCl}_{3}\right) \delta 142.49,139.36$, $137.54,136.21,129.72,129.25,126.68,125.97,119.76,117.85,33.03,33.16,31.70$, 28.99, 28.88, 22.66, 14.14. Anal. Calc'd. for $\mathrm{C}_{22} \mathrm{H}_{33} \mathrm{ClS}_{2}$ : C - 66.54; $\mathrm{H}-8.38 ; \mathrm{Cl}-8.93 ; \mathrm{S}$ - 16.15 Found, C - 66.62; H - 8.41; Cl - 8.95; S - 16.03.

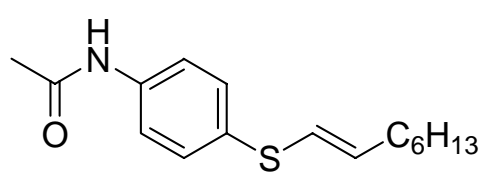

(E)-N-(4-(oct-1-enylthio)phenyl)acetamide (Table 2, entry 12): The general procedure was used to convert $N$-(4-mercaptophenyl)acetamide and $(E)$-1-iodooctene to the title product in 6 hours. Purification by flash chromatography (silica gel) (5\% ethyl acetate, $10 \%$ methanol and 3\% triethylamine in hexane as the eluent) gave the analytically pure product as a slightly yellow solid (541 mg, 97\% yield). ${ }^{1} \mathrm{H}$ NMR (400 MHz, $\left.\mathrm{CDCl}_{3}\right) \delta 8.04$ 
(s, 1H), 7.46-7.43 (d, J= 8.6 Hz; 2H), 7.25-7.22 (d, J= 8.6 Hz; 2H), 6.08-6.05 (d, J= 14.9 $\mathrm{Hz}$; $1 \mathrm{H}), 5.96-5.89(\mathrm{td}, \mathrm{J}=14.9,6.8 \mathrm{~Hz} ; 1 \mathrm{H}), 2.18-2.11(\mathrm{~m}, 5 \mathrm{H}), 1.42-1.25(\mathrm{~m}, 8 \mathrm{H}), 0.90-$ $0.87(\mathrm{t}, \mathrm{J}=7.0 \mathrm{~Hz} ; 3 \mathrm{H}) .{ }^{13} \mathrm{C} \mathrm{NMR}\left(100 \mathrm{MHz} \mathrm{CDCl}_{3}\right) \delta 168.77,136.94,136.39,131.37$, 129.54, 120.99, 120.61, 32.97, 31.54, 28.90, 28.69, 24.34, 22.53, 14.02. Anal. Calc'd. for $\mathrm{C}_{16} \mathrm{H}_{23} \mathrm{NOS}$ : C - 69.27; H - 8.36; N - 5.05; S - 11.56; Found, C - 69.44; H - 8.24; N 4.92; S - 11.35. m.p. $61-62^{\circ} \mathrm{C}$.

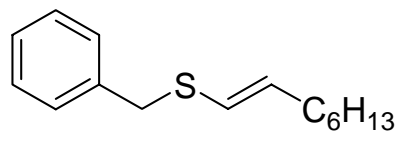

(E)-benzyl(oct-1-enyl)sulfane (Table 2, entry 13): The general procedure was used to convert benzyl mercaptan and $(E)$-1-iodooctene to the title product in 4 hours. Purification by flash chromatography (silica gel) (3\% triethylamine in hexane as the eluent) gave the analytically pure product as a colorless oil (464 mg, 99\% yield). ${ }^{1} \mathrm{H}$ NMR $\left(400 \mathrm{MHz}, \mathrm{CDCl}_{3}\right) \delta$ 7.31-7.30 (m, 4H), 7.25-7.23 (m, 1H), 5.91-5.87 (td, J= 15.0, $1.2 \mathrm{~Hz}$; $1 \mathrm{H}), 5.70-5.63(\mathrm{td}, \mathrm{J}=14.9,6.9 \mathrm{~Hz} ; 1 \mathrm{H}), 3.83(\mathrm{~s}, 2 \mathrm{H}), 2.05-2.00(\mathrm{dt}, J=7.8,6.8 \mathrm{~Hz} ; 2 \mathrm{H}$ ), 1.34-1.20 (m, 8H), 0.89-0.85 (t, J= 7.0 Hz; 3H). ${ }^{13} \mathrm{C}$ NMR $\left(100 \mathrm{MHz}, \mathrm{CDCl}_{3}\right) \delta 137.83$, 132.60, 128.75, 128.40, 126.95, 121.78, 37.56, 33.10, 31.62, 29.13, 28.62, 22.56, 14.06. Anal. Calc'd. for $\mathrm{C}_{15} \mathrm{H}_{22} \mathrm{~S}$ : C - 76.86; H - 9.46; S - 13.86; Found, C - 76.93; H - 9.41; S 13.96.

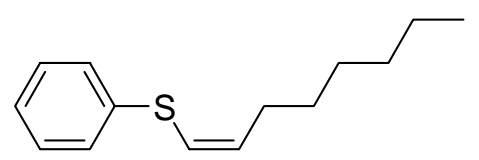

(Z)-1-phenylthio-1-octene (Table 3, entry 1): The general procedure was used to convert thiophenol and (Z)-1-iodooctene to the title product. Purification by flash chromatography (silica gel) (3\% triethylamine in hexanes as the eluent) gave the analytically pure product as a colorless liquid (425 mg, 96\% yield). ${ }^{1} \mathrm{H}$ NMR (400 MHz, $\left.\mathrm{CDCl}_{3}\right) \delta 7.35-7.27(\mathrm{~m}, 4 \mathrm{H}), 7.18(\mathrm{~m}, 1 \mathrm{H}), 6.17(\mathrm{td}, \mathrm{J}=9.20,1.36 ; 1 \mathrm{H}), 5.82(\mathrm{~m}, 1 \mathrm{H}), 2.25$ $(\mathrm{m}, \mathrm{J}=8.1,7.1,1.3 \mathrm{~Hz} ; 2 \mathrm{H}), 1.44-1.30(\mathrm{~m}, 8 \mathrm{H}), 0.89(\mathrm{t}, \mathrm{J}=6.8 \mathrm{~Hz} ; 3 \mathrm{H}) .{ }^{13} \mathrm{C}$ NMR $(100$ 
$\left.\mathrm{MHz}_{\mathrm{CDCl}}\right) \delta 136.54,133.72,128.89,128.67,126.02,122.47,31.67,29.12,28.99$, 28.89, 22.61, 14.08. Anal. Calc'd. for $\mathrm{C}_{14} \mathrm{H}_{20} \mathrm{~S}$ : C - 76.30; H - 9.15; S - 14.58 Found, C 76.03; H - 8.85; S - 14.32.

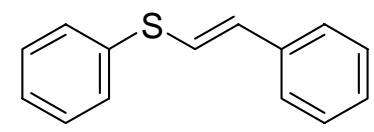

(E)-phenyl(styryl)sulfane (Table 3, entry 2): The general procedure was used to convert thiophenol and trans- $\beta$-iodostyrene to the title product. Purification by flash chromatography (neutral alumina) (pentane as the eluent) gave the analytically pure product as a colorless liquid (416 mg, 98\% yield). ${ }^{1} \mathrm{H}$ NMR $\left(400 \mathrm{MHz}, \mathrm{CDCl}_{3}\right) \delta 7.39(\mathrm{~m}$, 2H), 7.33-7.19 (m, 8H), $6.86(\mathrm{~d}, \mathrm{~J}=15.5 \mathrm{~Hz} ; 1 \mathrm{H}), 6.71$ (d, J= $15.5 \mathrm{~Hz} ; 1 \mathrm{H}) .{ }^{13} \mathrm{C}$ NMR (100 $\left.\mathrm{MHz} \mathrm{CDCl}_{3}\right) \delta 136.47,135.20,131.75,129.77,129.11,128.63,127.53,126.89,125.98$, 123.35. . Anal. Calc'd. for $\mathrm{C}_{14} \mathrm{H}_{12} \mathrm{~S}$ : C - 79.20; H - 5.70; S - 15.10 Found, C - 79.32; H 5.70; S - 15.04 .

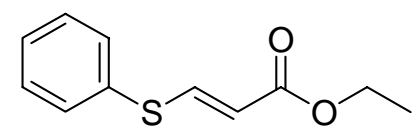

(E)-ethyl 3-(phenylthio)acrylate (Table 3, entry 3): The general procedure was used to convert thiophenol and $(E)$-3-iodopropenoate to the title product. Purification by flash chromatography (silica gel) (3\% triethylamine in hexane as the eluent) gave the analytically pure product as a light yellow oil (409 mg, 98\% yield). ${ }^{1} \mathrm{H}$ NMR (400 MHz, $\left.\mathrm{CDCl}_{3}\right) \delta$ 7.80-7.76 (d, J= 15.0 Hz; $\left.1 \mathrm{H}\right), 7.48-7.46(\mathrm{~m}, 4 \mathrm{H}), 7.42-7.38(\mathrm{~m}, 1 \mathrm{H})$, 5.67-5.64 $(\mathrm{d}, \mathrm{J}=15.1 \mathrm{~Hz} ; 1 \mathrm{H}), 4.18-4.13(\mathrm{q}, \mathrm{J}=7.1 \mathrm{~Hz} ; 2 \mathrm{H}), 1.27-1.23(\mathrm{t}, \mathrm{J}=7.1 \mathrm{~Hz} ; 3 \mathrm{H}) .{ }^{13} \mathrm{C} N M R$ $\left(100 \mathrm{MHz}, \mathrm{CDCl}_{3}\right) \delta 165.13,146.68,132.88,130.40,129.58,129.03,115.54,60.19$, 14.21. Anal. Calc'd. for $\mathrm{C}_{11} \mathrm{H}_{12} \mathrm{O}_{2} \mathrm{~S}$ : C - 63.43; H - 5.81 - S 15.40; Found, C - 63.50; H $5.81 ; \mathrm{S}-15.28$.

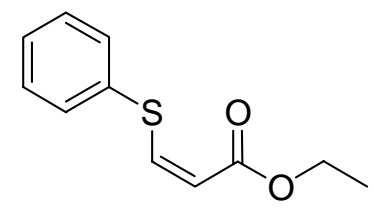


(Z)-ethyl 3-(phenylthio)acrylate (Table 3, entry 4): The general procedure was used to convert thiophenol and (Z)-3-iodopropenoate to the title product. Purification by flash chromatography (silica gel) (5\% ethyl acetate in mixture of 3\% triethylamine in hexane as the eluent) gave the analytically pure product as a slightly yellow oil (412 mg, 98\% yield). ${ }^{1} \mathrm{H}$ NMR (400 MHz, $\left.\mathrm{CDCl}_{3}\right) \delta$ 7.49-7.46 (m, 4H), 7.37-7.31 (m, 1H), 7.27-7.24 (d, $\mathrm{J}=10.0 \mathrm{~Hz} ; 1 \mathrm{H}), 5.92-5.89(\mathrm{~d}, \mathrm{~J}=10.0 \mathrm{~Hz} ; 1 \mathrm{H}), 4.26-4.21(\mathrm{q}, \mathrm{J}=7.1 \mathrm{~Hz} ; 2 \mathrm{H}), 1.33-1.29$ $(\mathrm{t}, \mathrm{J}=7.1 \mathrm{~Hz} ; 3 \mathrm{H}) .{ }^{13} \mathrm{C}$ NMR $\left(100 \mathrm{MHz}, \mathrm{CDCl}_{3}\right) \delta 166.32,149.54,135.97,130.86,129.17$, 128.02, 113.18, 60.13, 14.19. Anal. Calc'd. for $\mathrm{C}_{11} \mathrm{H}_{12} \mathrm{O}_{2} \mathrm{~S}$ : C - 63.43; $\mathrm{H}$ - 5.81; S - 15.40; Found, C - 63.66; H - 5.82; S - 15.24.

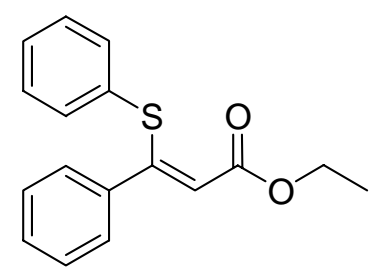

(Z)-methyl 3-phenyl-3-(phenylthio)acrylate (Table 3, entry 5): The general procedure was used to convert thiophenol and (Z)-methyl 3-iodo-3-phenylacrylate to the title product. Purification by flash chromatography (silica gel) (5\% ethyl acetate in mixture of $3 \%$ triethylamine in hexane as the eluent) gave the analytically pure product as a white solid (523 mg, 96\% yield). ${ }^{1} \mathrm{H}$ NMR (400 MHz, $\left.\mathrm{CDCl}_{3}\right) \delta$ 7.17-7.02 (m, 10H), $6.09(\mathrm{~s}, 1 \mathrm{H})$, $3.80(\mathrm{~s}, 3 \mathrm{H}) .{ }^{13} \mathrm{C}$ NMR $\left(100 \mathrm{MHz}, \mathrm{CDCl}_{3}\right) \delta 166.11,159.52,138.10,133.93,132.21$, 128.66, 128.35, 128.29, 127.67, 127.66, 115.64, 51.37. Anal. Calc'd. for $\mathrm{C}_{16} \mathrm{H}_{14} \mathrm{O}_{2} \mathrm{~S}$ : C 71.08; H - 5.22; S - 11.86; Found, C - 70.93; H - 5.21; S - 11.88. m.p. $72-73^{\circ} \mathrm{C}$.

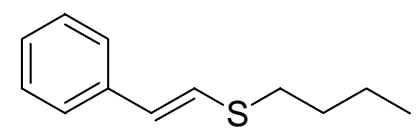

(E)-butyl(styryl)sulfane (Table 4, entry 1): The general procedure was used to convert butane-1-thiol and (E)-1-(2-iodovinyl)benzene to the title product in 4 hours. Purification by flash chromatography (silica gel) (3\% triethylamine in hexane as the eluent) gave the analytically pure product as a colorless oil (379 mg, 98\% yield). ${ }^{1} \mathrm{H}$ NMR (400 MHz, 
$\left.\mathrm{CDCl}_{3}\right) \delta$ 7.28-7.27 (m, 4H), 7.20-7.15 (m, 1H), 6.74-6.70 (d, J= 15.6 Hz; $\left.1 \mathrm{H}\right), 6.47-6.43$ $(\mathrm{d}, \mathrm{J}=15.6 \mathrm{~Hz} ; 1 \mathrm{H}), 2.81-2.77(\mathrm{t}, \mathrm{J}=7.3 \mathrm{~Hz} ; 2 \mathrm{H}), 1.71-1.63(\mathrm{~m}, 2 \mathrm{H}), 1.50-1.41(\mathrm{~m}, 2 \mathrm{H})$, 0.96-0.92 (t, J= $7.3 \mathrm{~Hz} ; 3 \mathrm{H}) .{ }^{13} \mathrm{C}$ NMR $\left(100 \mathrm{MHz}, \mathrm{CDCl}_{3}\right) \delta 137.10,128.55,126.67$, $126.53,125.37,125.31,32.23,31.46,21.89,13.63$. Anal. Calc'd. for $\mathrm{C}_{12} \mathrm{H}_{16} \mathrm{~S}$ : C - 74.94; H - 8.39; S - 16.67; Found, C - 75.08; H - 8.37; S - 16.88.

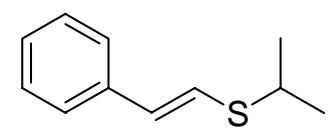

(E)-isopropyl(styryl)sulfane (Table 4, entry 2): The general procedure was used to convert propane-2-thiol and $(E)$-1-(2-iodovinyl)benzene to the title product in 4 hours. Purification by flash chromatography (silica gel) (5\% ethyl acetate in mixture of $3 \%$ triethylamine in hexane as the eluent) gave the analytically pure product as a colorless oil (320 mg, 89\% yield). ${ }^{1} \mathrm{H}$ NMR (400 MHz, $\left.\mathrm{CDCl}_{3}\right) \delta 7.30-7.26(\mathrm{~m}, 4 \mathrm{H}), 7.21-7.15(\mathrm{~m}$, $1 \mathrm{H}), 6.77-6.73(\mathrm{~d}, \mathrm{~J}=15.6 \mathrm{~Hz} ; 1 \mathrm{H}), 6.58-6.54(\mathrm{~d}, \mathrm{~J}=15.6 \mathrm{~Hz} ; 1 \mathrm{H}), 3.27-3.17$ (septet, $\mathrm{J}=$ $6.7 \mathrm{~Hz} ; 1 \mathrm{H}), 1.36-1.34(\mathrm{~d}, \mathrm{~J}=6.7 \mathrm{~Hz} ; 6 \mathrm{H}) .{ }^{13} \mathrm{C}$ NMR $\left(100 \mathrm{MHz}, \mathrm{CDCl}_{3}\right) \delta 137.04,128.78$, $128.55,126.89,125.54,124.03,36.79,23.36$. Anal. Calc'd. for $\mathrm{C}_{11} \mathrm{H}_{14} \mathrm{~S}$ : C - 74.10; H 7.91; S - 17.98; Found, C - 73.82; H - 7.91; S - 18.03.

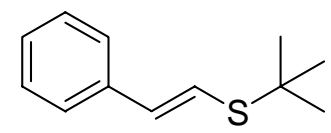

(E)-tert-butyl(styryl)sulfane (Table 4, entry 3): The general procedure was used to convert 2-methylpropane-2-thiol and $(E)$-1-(2-iodovinyl)benzene to the title product in 4 hours. Purification by flash chromatography (silica gel) (3\% triethylamine in hexane as the eluent) gave the analytically pure product as a colorless oil (309 mg, 80\% yield). ${ }^{1} \mathrm{H}$ NMR (400 MHz, $\left.\mathrm{CDCl}_{3}\right) \delta$ 7.34-7.27 (m, 4H), 7.22-7.18 (m, 1H), 6.89-6.85 (d, J= 15.4 $\mathrm{Hz} ; 1 \mathrm{H}), 6.73-6.69$ (d, J= $15.4 \mathrm{~Hz} ; 1 \mathrm{H}), 1.40$ (s, 9H). ${ }^{13} \mathrm{C}$ NMR (100 MHz, $\left.\mathrm{CDCl}_{3}\right)$ $\delta 136.98,131.91,128.55,127.19,125.82,121.99,44.28,30.97$. Anal. Calc'd. for $\mathrm{C}_{12} \mathrm{H}_{16} \mathrm{~S}$ : C - 74.94; H - 8.39; S - 16.67; Found, C - 74.73; H - 8.29; S - 16.51. 


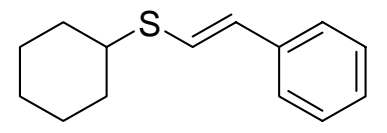

(E)-cyclohexyl(styryl)sulfane (Table 4, entry 4): The general procedure was used to convert cyclohexanethiol and $\beta$-iodostyrene to the title product. Purification by flash chromatography (silica gel) (3\% triethylamine in hexanes as the eluent) gave the analytically pure product as a light yellow liquid (417 mg, 95\% yield). ${ }^{1} \mathrm{H}$ NMR (400 MHz, $\left.\mathrm{CDCl}_{3}\right) \delta 7.28(\mathrm{~m}, 4 \mathrm{H}), 7.18(\mathrm{~m}, 1 \mathrm{H}), 6.76(\mathrm{~d}, \mathrm{~J}=15.6 \mathrm{~Hz}, 1 \mathrm{H}), 6.56(\mathrm{~d}, \mathrm{~J}=15.6 \mathrm{~Hz} ; 1 \mathrm{H})$, $2.98(\mathrm{~m}, 1 \mathrm{H}), 2.05(\mathrm{~m}, 2 \mathrm{H}), 1.79(\mathrm{~m}, 2 \mathrm{H}), 1.63(\mathrm{~m}, 1 \mathrm{H}), 1.45-1.28(\mathrm{~m}, 5 \mathrm{H}) .{ }^{13} \mathrm{C}$ NMR $(100$ $\left.\mathrm{MHz}, \mathrm{CDCl}_{3}\right) \delta 137.11,128.55,126.83,125.52,124.01,45.27,35.57,25.99,25.64$. Anal. Calc'd. for $\mathrm{C}_{14} \mathrm{H}_{18} \mathrm{~S}$ : C - 77.01; H - 8.31; S - 14.68 Found, C - 76.88; H - 8.32; S 14.75 .

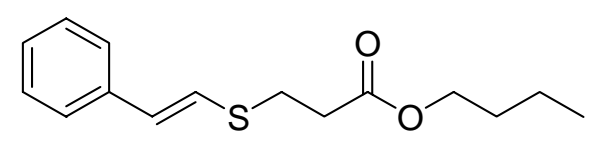

(E)-butyl 3-(styrylthio)propanoate (Table 4, entry 5): The general procedure was used to convert butyl 3-mercaptopropanoate and (E)-1-(2-iodovinyl)benzene to the title product in 4 hours. Purification by flash chromatography (silica gel) (3\% triethylamine in hexane as the eluent) gave the analytically pure product as a colorless oil (521 mg, 98\% yield). ${ }^{1} \mathrm{H}$ NMR (400 MHz, $\left.\mathrm{CDCl}_{3}\right) \delta$ 7.29-7.28(m, 4H), 7.22-7.17 (m, 1H), 6.70-6.66 (d, $J=15.5 \mathrm{~Hz} ; 1 \mathrm{H}), 6.53-6.50(\mathrm{~d}, \mathrm{~J}=15.5 \mathrm{~Hz} ; 1 \mathrm{H}), 4.12-4.08(\mathrm{t}, \mathrm{J}=6.7 \mathrm{~Hz} ; 2 \mathrm{H}), 3.07-3.04(\mathrm{t}$, $J=7.3 \mathrm{~Hz} ; 2 \mathrm{H}), 2.72-2.68(\mathrm{t}, \mathrm{J}=7.3 \mathrm{~Hz} ; 2 \mathrm{H}), 1.64-1.57(\mathrm{~m}, 2 \mathrm{H}), 1.42-1.32(\mathrm{~m}, 2 \mathrm{H}), 0.94-$ 0.90 (t, J= 7.3 Hz; 3H). ${ }^{13} \mathrm{C}$ NMR $\left(100 \mathrm{MHz} \mathrm{CDCl}_{3}\right) \delta 171.66,136.70,128.57,128.31$, 127.02, 125.53, 123.82, 64.66, 34.62, 30.53, 27.60, 19.04, 13.63. Anal. Calc'd. for $\mathrm{C}_{15} \mathrm{H}_{20} \mathrm{O}_{2} \mathrm{~S}$ : C - 68.14; H - 7.62; S - 12.13; Found, C - 67.98; H - 7.60; S - 12.26.

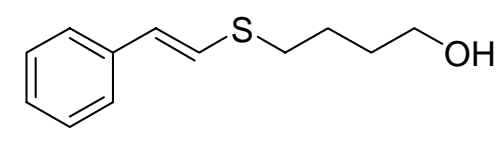


(E)-4-(styrylthio)butan-1-ol (Table 4 entry 6): The general procedure was used to convert 4-mercapto-1-butanol and $\beta$-iodostyrene to the title product. Purification by flash chromatography (silica gel) (3\% triethylamine in ethyl acetate as the eluent) gave the analytically pure product as a colorless liquid (403 mg, 97\% yield). ${ }^{1} \mathrm{H}$ NMR (400 MHz, $\left.\mathrm{CDCl}_{3}\right) \delta 7.28(\mathrm{~d}, \mathrm{~J}=4.4 \mathrm{~Hz} ; 4 \mathrm{H}), 7.17(\mathrm{~m}, 1 \mathrm{H}), 6.70(\mathrm{~d}, \mathrm{~J}=15.6 \mathrm{~Hz} ; 1 \mathrm{H}), 6.46(\mathrm{~d}, \mathrm{~J}=15.6$ $\mathrm{Hz} ; 1 \mathrm{H}), 3.67$ (t, J=6.3 Hz; 2H), 2.83 (t, J= 7.0 Hz; 2H), 1.80-1.63 (m, 4H), 1.53 (broad s, 1H). ${ }^{13} \mathrm{C} \mathrm{NMR}\left(100 \mathrm{MHz}, \mathrm{CDCl}_{3}\right) \delta 136.97,128.57,127.02,126.80,125.42,124.90$, 62.27, 32.37, 31.62, 25.76. IR (KBr): 3355 (s,br); 3074 (m); 3020 (m); 2936 (s); 2871 (s); 1944 (w); 1594 (s); 1568 (s); 1446 (s); 1056 (s); 937 (s); 736 (s); 691 (s). Anal. Calc'd. for $\mathrm{C}_{12} \mathrm{H}_{16} \mathrm{OS}$ : C - 69.19; H - 7.74; S - 15.39 Found, C - 69.18, H - 7.66; 15.51.

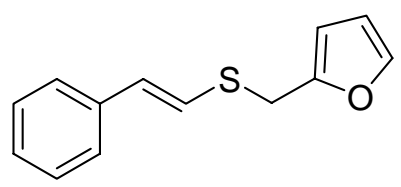

(E)-2-(styrylthiomethyl)furan (Table 4 entry 7): The general procedure was used to convert furfuryl mercaptan and $\beta$-iodostyrene to the title product. Purification by flash chromatography (silica gel) (5\% ethyl acetate in a 3\% triethylamine in hexanes solution as the eluent) gave the analytically pure product as a colorless liquid (419 $\mathrm{mg}, 97 \%$ yield). ${ }^{1} \mathrm{H}$ NMR $\left(400 \mathrm{MHz}, \mathrm{CDCl}_{3}\right) \delta 7.36(\mathrm{dd}, \mathrm{J}=1.8,0.8 \mathrm{~Hz} ; 1 \mathrm{H}), 7.27(\mathrm{~m}, 4 \mathrm{H}), 7.18(\mathrm{~m}$, $1 \mathrm{H}), 6.72(\mathrm{~d}, \mathrm{~J}=15.9 \mathrm{~Hz} ; 1 \mathrm{H}), 6.55$ (d, J= $15.9 \mathrm{~Hz} ; 1 \mathrm{H}), 6.31$ (dd, J= 3.3, $1.9 \mathrm{~Hz} ; 1 \mathrm{H})$, 6.24 (dd, J=3.5, $0.7 \mathrm{~Hz} ; 1 \mathrm{H}$ ), $3.98(\mathrm{~s}, 2 \mathrm{H}) .{ }^{13} \mathrm{C}$ NMR $\left(100 \mathrm{MHz}, \mathrm{CDCl}_{3}\right) \delta 150.79,142.32$, 136.74, 128.64, 128.57, 127.08, 125.63, 123.72, 110.51, 107.84, 29.63. Anal. Calc'd. for $\mathrm{C}_{13} \mathrm{H}_{12} \mathrm{OS}$ : C - 72.19; H - 5.59; S - 14.82 Found, C - 72.01; H - 5.51; S - 14.98.

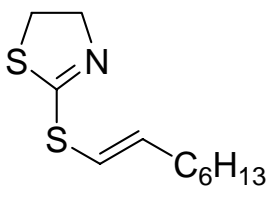

(E)-2-(oct-1-enylthio)-4,5-dihydrothiazole (Table 5, entry 1): The general procedure was used to convert 2-mercaptothiazoline and $(E)$-1-iodooctene to the title product in 12 hours. Purification by flash chromatography (silica gel) (5\% ethyl acetate in mixture of 
$3 \%$ triethylamine in hexane as the eluent) gave the analytically pure product as a colorless oil (405 mg, 88\% yield). ${ }^{1} \mathrm{H}$ NMR (400 MHz, $\left.\mathrm{CDCl}_{3}\right) \delta 6.45-6.40$ (td, $\mathrm{J}=15.2,1.3$ $\mathrm{Hz} ; 1 \mathrm{H}), 6.07-6.00$ (td, J= 15.2, 7.0 Hz; 1H), 4.26-4.22 (t, J=8.0 Hz; 2H), 3.39-3.35 (t, J= $8.0 \mathrm{~Hz} ; 2 \mathrm{H}), 2.19-2.14(\mathrm{dt}, \mathrm{J}=8.0,7.0 \mathrm{~Hz} ; 2 \mathrm{H}), 1.45-1.25(\mathrm{~m}, 8 \mathrm{H}), 0.90-0.86(\mathrm{t}, \mathrm{J}=7.0$ $\mathrm{Hz} ; 3 \mathrm{H}) .{ }^{13} \mathrm{C}$ NMR $\left(100 \mathrm{MHz}, \mathrm{CDCl}_{3}\right) \delta 165.78,139.37,116.85,64.63,35.01,33.00$, 31.51, 28.66, 28.56, 22.48, 13.98. Anal. Calc'd. for $\mathrm{C}_{11} \mathrm{H}_{19} \mathrm{NS}_{2}$ : C - 57.59; H - 8.35; N 6.11; S - 27.95; Found, C - 57.71; H - 8.35; N - 6.06; S - 28.00.

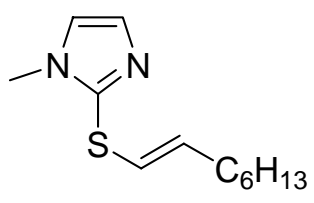

(E)-1-methyl-2-(oct-1-enylthio)-1H-imidazole (Table 5, entry 2): The general procedure was used to convert 2-mercapto-1-methylimidazole and $(E)$-1-iodooctene to the title product in 24 hours. Purification by flash chromatography (neutral alumina) (10\% ethyl acetate in mixture of $3 \%$ triethylamine in hexane as the eluent) gave the analytically pure product as a colorless oil (448 mg, 99\% yield). ${ }^{1} \mathrm{H}$ NMR (400 MHz, $\left.\mathrm{CDCl}_{3}\right) \delta 7.07(\mathrm{~d}, \mathrm{~J}=1.2 \mathrm{~Hz} ; 1 \mathrm{H}), 6.97(\mathrm{~d}, \mathrm{~J}=1.2 \mathrm{~Hz} ; 1 \mathrm{H}), 6.11-6.08$ (td, J= 15.0, $1.3 \mathrm{~Hz}$; $1 \mathrm{H}), 5.81-5.74(\mathrm{td}, \mathrm{J}=15.0,7.0 \mathrm{~Hz} ; 1 \mathrm{H}), 3.63(\mathrm{~s}, 3 \mathrm{H}), 2.12-2.07(\mathrm{dt}, J=8.0,7.0 \mathrm{~Hz} ; 2 \mathrm{H})$, $1.38-1.25(\mathrm{~m}, 8 \mathrm{H}), 0.89-0.85(\mathrm{t}, \mathrm{J}=7.0 \mathrm{~Hz} ; 3 \mathrm{H}) \cdot{ }^{13} \mathrm{C}$ NMR $\left(100 \mathrm{MHz}, \mathrm{CDCl}_{3}\right) \delta 139.51$, 134.88, 129.43, 122.65, 118.84, 33.27, 32.77, 31.45, 28.78, 28.59, 22.41, 13.91. Anal. Calc'd. for $\mathrm{C}_{12} \mathrm{H}_{20} \mathrm{~N}_{2} \mathrm{~S}$ : C - 64.24; H - 8.98; N - 12.49; S - 14.29; Found, C - 64.09; H 9.02; N - 12.22; S - 14.37.

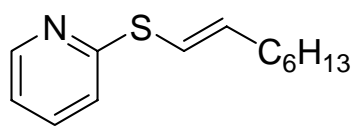

(E)-2-(oct-1-enylthio)pyridine (Table 5, entry 3): The general procedure was used to convert pyridine-2-thiol and $(E)$-1-iodooctene to the title product in 24 hours. Purification by flash chromatography (silica gel) (3\% triethylamine in hexane as the eluent) gave the analytically pure product as a colorless oil (442 mg, 99\% yield). ${ }^{1} \mathrm{H}$ NMR (400 MHz, 
$\left.\mathrm{CDCl}_{3}\right) \delta 8.44-8.42(\mathrm{ddd}, \mathrm{J}=5.0,2.0,1.0 \mathrm{~Hz} ; 1 \mathrm{H}), 7.52-7.47(\mathrm{dt}, \mathrm{J}=7.5,2.0 \mathrm{~Hz} ; 1 \mathrm{H})$, 7.17-7.15 (m, 1H), 7.00-6.97 (ddd, J= 7.5, 4.8, 1.2 Hz; 1H), 6.56-6.52 (td, J= 15.3, 1.4 $\mathrm{Hz} ; 1 \mathrm{H}), 6.11-6.04$ (td, J= 15.2, 7.0 Hz; 1H), 2.25-2.19 (dt, J= 8.0, 7.0 Hz; 2H), 1.48-1.26 $(\mathrm{m}, 8 \mathrm{H}), 0.91-0.87(\mathrm{t}, \mathrm{J}=7.0 \mathrm{~Hz} ; 3 \mathrm{H}) .{ }^{13} \mathrm{C}$ NMR $\left(100 \mathrm{MHz}, \mathrm{CDCl}_{3}\right) \delta$ 159.34, 149.52, 137.69, 136.16, 121.21, 119.59, 117.67, 33.21, 31.54, 28.84, 28.71, 22.50, 13.99. Anal. Calc'd. for $\mathrm{C}_{13} \mathrm{H}_{19} \mathrm{NS}$ : C - 70.54; $\mathrm{H}$ - 8.65; N - 6.33; S - 14.49; Found, C - 70.61; H - 8.67; N - 6.55; S - 14.34 .

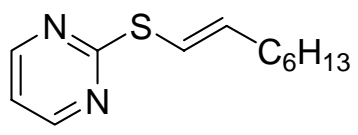

(E)-2-(oct-1-enylthio)pyrimidine (Table 5, entry 4): The general procedure was used to convert pyrimidine-2-thiol and $(E)$-1-iodooctene to the title product in 24 hours. Purification by flash chromatography (silica gel) (5\% ethyl acetate in mixture of $3 \%$ triethylamine in hexane as the eluent) gave the analytically pure product as a slightly yellow oil (431 mg, 97\% yield). ${ }^{1} \mathrm{H}$ NMR (400 MHz, $\left.\mathrm{CDCl}_{3}\right) \delta 8.52-8.51$ (d, J=4.8 Hz; $\left.2 \mathrm{H}\right)$, 6.99-6.96 (t, J= 4.8 Hz; 1H), 6.74-6.70 (td, J= 15.5, $1.3 \mathrm{~Hz} ; 1 \mathrm{H}), 6.06-5.98(\mathrm{td}, \mathrm{J}=15.5$, $7.0 \mathrm{~Hz} ; 1 \mathrm{H}), 2.25-2.19(\mathrm{dt}, \mathrm{J}=7.8,7.0 \mathrm{~Hz} ; 2 \mathrm{H}), 1.48-1.26(\mathrm{~m}, 8 \mathrm{H}), 0.90-0.87(\mathrm{t}, \mathrm{J}=7.0$ $\mathrm{Hz} ; 3 \mathrm{H}) .{ }^{13} \mathrm{C}$ NMR $\left(100 \mathrm{MHz}, \mathrm{CDCl}_{3}\right) \delta 171.59,157.17,135.56,117.61,116.57,33.14$, 31.47, 28.75, 28.61, 22.42, 13.91. Anal. Calcd. for $\mathrm{C}_{12} \mathrm{H}_{18} \mathrm{~N}_{2} \mathrm{~S}$ : C - 64.82; H - 8.16; N 12.60; S - 14.42; Found, C - 64.71; H - 8.07; N - 12.44; S - 14.22.

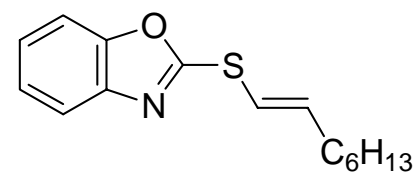

(E)-2-(oct-1-enylthio)benzo[d]oxazole (Table 5, entry 5): The general procedure was used to convert 2-mercaptobenzoxazole and $(E)$-1-iodooctene to the title product in 8 hours. Purification by flash chromatography (silica gel) (3\% triethylamine in hexane as the eluent) gave the analytically pure product as a colorless oil (520 mg, 99\% yield). ${ }^{1} \mathrm{H}$ NMR (400 MHz, $\left.\mathrm{CDCl}_{3}\right) \delta$ 7.63-7.60 (m, 1H), 7.43-7.41 (m, 1H), 7.29-7.20 (m, 2H), 6.55- 
$6.52(\mathrm{td}, \mathrm{J}=15.2,1.3 \mathrm{~Hz} ; 1 \mathrm{H}), 6.20-6.13(\mathrm{td}, \mathrm{J}=15.2,7.0 \mathrm{~Hz} ; 1 \mathrm{H}), 2.27-2.21$ (dt, J= 8.3, $7.0 \mathrm{~Hz} ; 2 \mathrm{H}), 1.49-1.26(\mathrm{~m}, 8 \mathrm{H}), 0.91-0.88(\mathrm{t}, \mathrm{J}=7.0 \mathrm{~Hz} ; 3 \mathrm{H}) .{ }^{13} \mathrm{C}$ NMR $\left(100 \mathrm{MHz}, \mathrm{CDCl}_{3}\right)$ $\delta 163.64,151.73,141.88,140.17,124.28,123.89,118.54,114.32,109.83,33.18,31.56$, 28.71, 28.62, 28.53, 14.02. Anal. Calc'd. for $\mathrm{C}_{15} \mathrm{H}_{19} \mathrm{NOS}$ : C - 68.93; H - 7.33; N - 5.36; S - 12.27; Found, C - 69.07; H - 7.25; N - 5.25; S - 12.00.

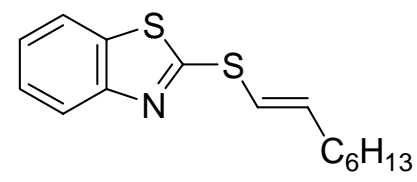

(E)-2-(oct-1-enylthio)benzo[d]thiazole (Table 5, entry 6): The general procedure was used to convert 2-mercaptobenzothiazole and $(E)$-1-iodooctene to the title product in 8 hours. Purification by flash chromatography (silica gel) (3\% triethylamine in hexane as the eluent) gave the analytically pure product as a colorless oil (549 mg, $98 \%$ yield). ${ }^{1} \mathrm{H}$ NMR (400 MHz, $\left.\mathrm{CDCl}_{3}\right) \delta 7.87-7.85(\mathrm{~d}, \mathrm{~J}=8.2 \mathrm{~Hz} ; 1 \mathrm{H}), 7.72-7.70(\mathrm{~d}, \mathrm{~J}=7.9 \mathrm{~Hz} ; 1 \mathrm{H})$, 7.40-7.36 (m, 1H), 7.26-7.23 (m, 1H), 6.47-6.43 (td, J= 14.9, $1.2 \mathrm{~Hz} ; 1 \mathrm{H}), 6.29-6.22$ (td, $J=14.9,6.9 \mathrm{~Hz} ; 1 \mathrm{H}), 2.25-2.19(\mathrm{dt}, \mathrm{J}=8.0,6.8 \mathrm{~Hz} ; 2 \mathrm{H}), 1.49-1.25(\mathrm{~m}, 8 \mathrm{H}), 0.91-0.88$ (t, $J=7.1 \mathrm{~Hz} ; 3 \mathrm{H}) .{ }^{13} \mathrm{C}$ NMR $\left(100 \mathrm{MHz}, \mathrm{CDCl}_{3}\right) \delta 167.59,153.66,143.31,135.04,125.93$, 123.96, 121.54, 120.68, 116.19, 33.04, 31.46, 28.63, 28.39, 22.47, 13.97. Anal. Calc'd. for $\mathrm{C}_{15} \mathrm{H}_{19} \mathrm{NS}_{2}$ : C - 64.93; $\mathrm{H}-6.90 ; \mathrm{N}-5.05 ; \mathrm{S}-23.11$; Found, C - 65.20; H - 6.96; N 4.79; S - 23.00.

References:

(1) Piers, E.; Wong, T.; Coish, P. D.; Rogers C. Can. J. Chem. 1994, 72, 1816-1819.

(2) Han, C.; Shen, R. C.; Su, S.; Porco J. A. Org Lett. 2004, 6, 27-30.

(3) Lee, K.; Wiemer, D. F. Tetrahedron Lett. 1993, 34, 2433-2436.

(4) Brown, H. C.; Subrahmanyam, C.; Hamaoka, T.; Ravindran, N.; Bowman, D. H.; Misumi, S.; Unni, M. K.; Somayaji, V.; Bhat, N. G. J. Org. Chem. 1989, 54, 6068-6075.

(5) Bates, C. G.; Saejueng, P.; Murphy, J. M.; Venkataraman, D. Org. Lett. 2002, 4, 4727-4729.

(6) Kubas, G. J. Inorg. Synth. 1979, 19, 90-92.

(7) Bates, C. G.; Saejueng, P.; Venkataraman, D. Org. Lett. 2004, 6, 1441-1444. 